\title{
Research on the Wireless Sensor Network Routing Model Based on Fluid Mechanics
}

\author{
Ming $\mathrm{Li}^{1,2, \mathrm{a}^{*}}$, Huanyan Qian ${ }^{1, \mathrm{~b}}$ and Jiang $\mathrm{Xu}^{3, \mathrm{c}}$ \\ ${ }^{1}$ School of Computer Science and Engineering, Nanjing University of Science and Technology, \\ Nanjing, 210094, China \\ ${ }^{2}$ School of Business, Hohai University, Nanjing, 211100, China \\ ${ }^{3}$ Changshu Institute of Technology, 215500, China \\ am@hhu.edu.cn, bhyqian@njust.edu.cn, ${ }^{\mathrm{a}}$ skydestinyx@gmail.com
}

Keywords: Wireless sensor network; Routing model; Fluid mechanics; N-S equation.

\begin{abstract}
A fluid is divided into the viscous fluid and the non-viscous fluid. In the fluid mechanics, elemental volumes of fluid move in a flow line with frictional resistance among them. It is similar to the process in a wireless sensor network in which data packets, starting from the source node, are forwarded by a series of intermediate nodes in the routing direction, and finally reach the sink node. In this process, data packets also have conflicts and collisions, similar to the frictional resistance. Through a comparative analysis, on the basis of N-S equation of vicious fluid motion, the paper builds a viscous-fluid-based wireless sensor network routing model and proposes the density function of wireless sensor network. Through the simulation analysis on the model, the paper finds that the load flow in the wireless sensor network routing model based on vicious fluid is orderly and even.
\end{abstract}

\section{Introduction}

The fluid mechanics is a branch of mechanics studying the relations between and laws of fluid motion and applied force from a macroscopic perspective with the fluid as the object. The subject focuses on the laws of equilibrium and motion of the fluid under external forces [1]. The fluid includes the liquid and the gas, with the most significant property of flowing easily. The real fluid has the viscosity showing as producing frictional resistance in the internal or on the contact interface of fluid in the flow process [2]. In the fluid mechanics, the fluid is generally divided into the real liquid (also called viscous fluid) and the neglected ideal fluid (also known as inviscid fluid), and the former is further divided into the Newtonian fluid and the non-Newtonian fluid. The ideal fluid and the Newtonian fluid are the research objects of fluid mechanics, while the non-Newtonian fluid is the research object of rheological mechanics [3, 4].

In the fluid mechanics, the flow line means the motion track of a fluid particle in the space, showing the spatial positions of the fluid particle at different instants. The flow line refers to an imaginary smooth curve in an instantaneous flow field, in which each point's tangent line and its velocity vector coincide. The flowing of elemental volumes can be seen clearly by drawing many flow lines at the same instant.

According to the definition of flow line, it's possible to make the flow line passing point $M_{1}$ at the instant of $t_{0}$ in the flow field. Make velocity vector $v_{1}$ of fluid particle at point $M_{1}$, make velocity vector $v_{2}$ of instant fluid particle $M_{2}$ which is $\Delta s$ away from point $M_{1}$ along the direction of $v_{1}$, and then make velocity vector $v_{3}$ of instant fluid particle $M_{3}$ which is $\Delta s$ away from point $M_{2}$ along the direction of $v_{2}$, and finally get a broken line $M_{1} M_{2} M_{3} \ldots \ldots$ in the same way. Make $\Delta s$, the distance between points approach zero, and then the broken line shall become a smooth curve denoted as the flow line, as shown in Fig. 1. 


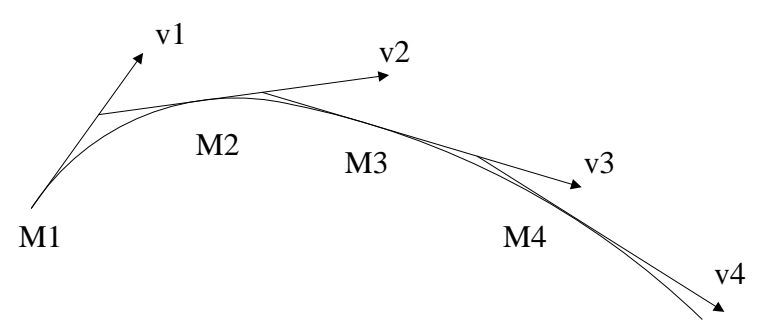

Figure 1. Flow Line

Except the special circumstances like the stationary point in a flow over a body, the flow line can only be a smooth curve without any intersection or turning. Considering the data packets in a sensor network as the particles in a field at different instants, and then according to the positions of data packets, the flow of data packets can be considered as a smooth curve, from which we can find the routing of data packets in a wireless sensor network.

\section{The Analysis on Wireless Sensor Network Routing}

In the process of transmitting data packets in a wireless sensor network, data packets are forwarded by a series of intermediate nodes in the routing direction and finally reach the sink node[5]. The process is similar to the process of fluid mechanics in which the fluid flows from a source point, moves along a flow line and finally reaches a sink point. Therefore, it's reasonable to make an analogy between fluid model and data packet flow in wireless sensor network. The analysis model based on inviscid fluid neglects the viscosity of fluid and then the frictional resistance produced in the internal of fluid during flowing, and thus neglects the conflicts and collisions of data packets in sensor network. The simulation process shows that the solution of ideal fluid generally doesn't consist with the results of actual flow experiment. For instance, in the simulation process of single source node, loads flow in disordered directions in some regions in the network, and in the simulation process of multiple source nodes, loads around the cluster-head node show messy flow directions. The reason is that the analysis model based on inviscid fluid neglects the viscosity of fluid and thus neglects the influence of internal frictional resistance of fluid. Similarly, in the routing process of wireless sensor network, it will neglect the conflicts and collisions of data packets in the transmission process in the sensor network. In fact, each sensor node in network may receive data packets from different directions or a lot of data packets from the same direction, so the conflicts and collisions of data packets received are inevitable. Therefore, the viscous fluid model is more applicable to the modeling of wireless sensor network routing. The next section makes the modeling and analysis of sensor network routing with a viscous fluid model.

\section{N-S Equation-based Wireless Sensor Network Routing Model}

The difference between real fluid and ideal fluid is the viscosity. The real fluid has the viscosity and thus is also known as the viscous fluid. The fluid's property resisting the relative motions among elemental volumes is called the viscosity, showing as the friction between fluids. In the real fluid with viscosity, the situation is much more complicated that the differential equation of ideal fluid can't be applied to the flow of real viscous fluid. The sensor network taking no account of data packet conflict can be analyzed with the Euler equation based on inviscid fluid; while, when taking consideration of conflicts and collisions of data packets in sensor network, the data packets shall be analyzed with the Navier-Stokes equation. The Navier-Stokes equation has a viscosity term added compared with the differential equation of ideal fluid, and thus is a more complicated nonlinear partial differential equation.

The N-S equation, short for the Navier-Stokes equation, is a basic equation analyzing the relationship between real fluid motion and force[6]. For the purpose of the flow of data packets in wireless sensor network, the paper uses the N-S equation to analyze the flow of loads in the sensor 
network. Therefore, the paper improves the partial differential equation of load flow field with the N-S equation.

In the fluid mechanics, the viscous fluid motion refers to the flow including phenomena such as friction, heat conduction and mass diffusion. The motion of transport phenomena is interpreted as the diffusion and flow phenomena of data packets in the wireless sensor network. The following is the equation for the incompressible flow with the constant viscosity.

$$
\frac{d u}{d t}=f-\frac{1}{\rho} \nabla p+v \nabla^{2} u .
$$

Eq. 1 gives the vector form of T-S equation. The paper only explores the two-dimensional motion, so only selects the two-dimensional representation of T-S equation. The components expressions in the rectangular coordinates system are as follows:

$$
\begin{aligned}
& \frac{d u}{d t}=f_{x}-\frac{1}{\rho} \frac{\partial p}{\partial x}+v\left(\frac{\partial^{2} u}{\partial x^{2}}+\frac{\partial^{2} u}{\partial y^{2}}\right) \\
& \frac{d v}{d t}=f_{y}-\frac{1}{\rho} \frac{\partial p}{\partial y}+v\left(\frac{\partial^{2} v}{\partial x^{2}}+\frac{\partial^{2} v}{\partial y^{2}}\right)
\end{aligned} .
$$

where $v$ is the coefficient of viscosity, $\rho$ is the flow lien distribution density, $f_{x}$ and $f_{y}$ are component forces per unit in the direction of $\mathrm{x}$ and the direction of $\mathrm{y}$, respectively. Make an equation conversion by setting $v$ as 1 (because $v$ is a constant, to paper let it be 1 for simplicity), and then get

$$
\frac{d u}{d t}-\nabla^{2} u=f-\frac{1}{\rho} \nabla p
$$

Eq. 3 is used to analyze the relation between the fluid's motion and force, where $u$ is the velocity of fluid, $f$ is the force on the fluid, and $p$ is the static pressure of fluid.

$$
\frac{d u}{d t}-\nabla^{2} u=f .
$$

In Eq. $4, \nabla^{2}$ is the Laplace operator (quadratic differential operator) [7,8]. The analysis on $f$ : in the sensor network, the paper shall consider it as the flow intensity of data packets, because many data packets are distributed near the source node, the flow intensity of load in this region is high. Therefore, get the N-S equation of load flow field in the sensor network, as shown in Eq. 5:

$$
\frac{d \vec{G}}{d t}-\nabla^{2} \vec{G}=\lambda \rho
$$

From Eq. 6,

$$
\rho(x, y)=\beta \sigma(x, y) \text {. }
$$

it can be obtained that

$$
\frac{d \vec{G}}{d t}-\nabla^{2} \vec{G}=\lambda \beta \sigma=\varepsilon \sigma
$$

where $\sigma$ is the density distribution function of source node and $\beta$ is a constant.

In mathematics, the $\mathrm{N}-\mathrm{S}$ equation is an oval-shaped second-order partial differential equation of which the boundary condition should be a Dirichlet or Neuman condition in the closed boundary $[9,10]$, basing on which as well as the boundary condition, we can get the corresponding load flow field $\vec{G}$. To prevent the fluid from flowing out of boundaries. $\vec{G}$ is 0 in boundaries, as shown in Eq. 8 . 
$\vec{G} \cdot \vec{n}=0$ (boundary condition).

Therefore, the sensor network load flow filed based on the viscous fluid model can be described with Eq. 9.

$$
\left\{\begin{array}{l}
\frac{d \vec{G}}{d t}-\nabla^{2} \vec{G}=\varepsilon \sigma \\
\vec{G} \cdot \vec{n}=0 \text { (boundary condition) }
\end{array} .\right.
$$

Therefore, basing on the viscous fluid model, the paper gets the description on the new load flow field of sensor network. Different from the one based on the inviscid fluid model, the load flow field of sensor network in the paper has taken the possible conflicts and collisions of data packets along the load flow line into consideration.

The analysis above shows that to simulate the load flow field of sensor network, the researcher should first determine the distribution of source and destination nodes of sensor network, next determine the distribution of field intensity on each node, and then further determine the density distribution function of source node on each node, in order to determine the load flow field and routing of sensor network through the source-node density function.

\section{Model Simulation and Analysis}

Single-source-node Scene Simulation. The research uses Matlab for the solution of a scene of single source node, and gets the load flow field shown in Fig. 2.
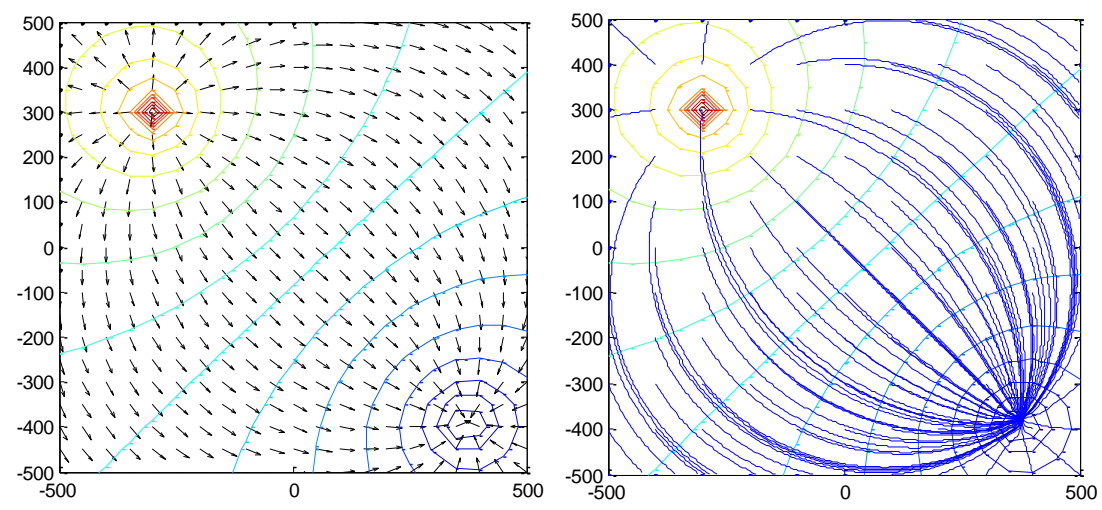

Figure 2. Single Source Node Load Flow Field

It can be seen from Fig. 2 that the load flow field built on the basis of viscous fluid model explains the flow of load in wireless sensor network well. The source node is in the position of $(-300,300)$ and the destination node is in the position of $(400,-400)$, consistent with the original definitions. It also can be seen that there is no disorderly flow of load in the sensor network and data packets flow from the position of source node (upper left corner) to the position of destination node (lower right corner) orderly in the load flow direction.

Fig. 3 shows the comparison of single-source-node load flow fields based on inviscid fluid and viscous fluid.

Fig. 3 (a) shows a sensor network load flow field obtained from an inviscid fluid model, and Fig. 3 (b) is a sensor network load flow field obtained from a viscous fluid model. It can be seen in Fig. 3 (a) that loads flow in messy directions and the position of source node has a large deviation from the practical position; while, the sensor network load flow field obtained from viscous fluid model solves the problems very well, in which the source node transmits data packets orderly to the destination node along the load flow direction. 


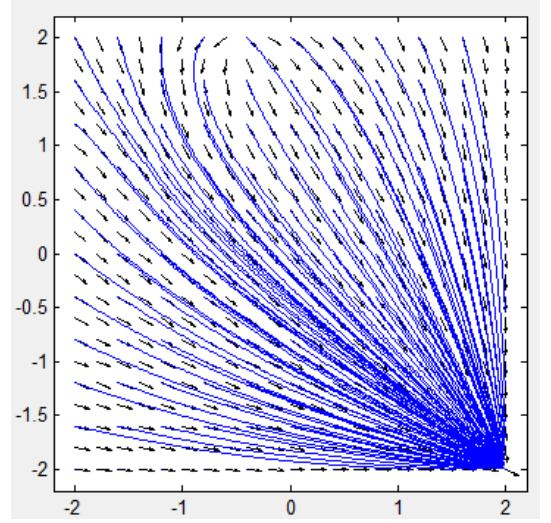

(a) Load Flow Field of Inviscid Fluid

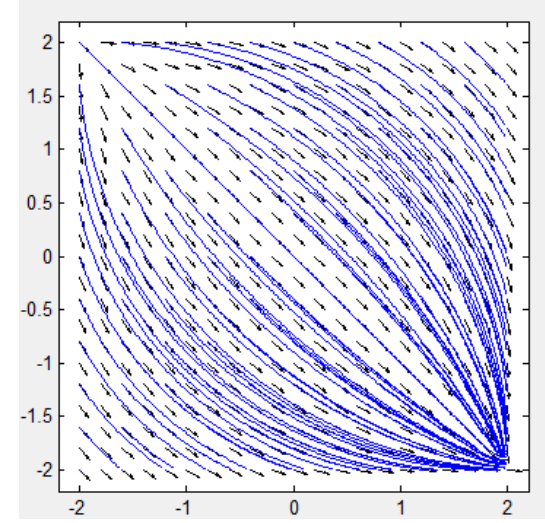

(b) Load Flow Field of Viscous Fluid

Figure 3. Comparison of Single Source Node

Multiple-source-node Scene Simulation. Fig. 4 shows a simulation of multiple-source-node scene with Matlab, in which the arrow points to the load flow directions at the point.
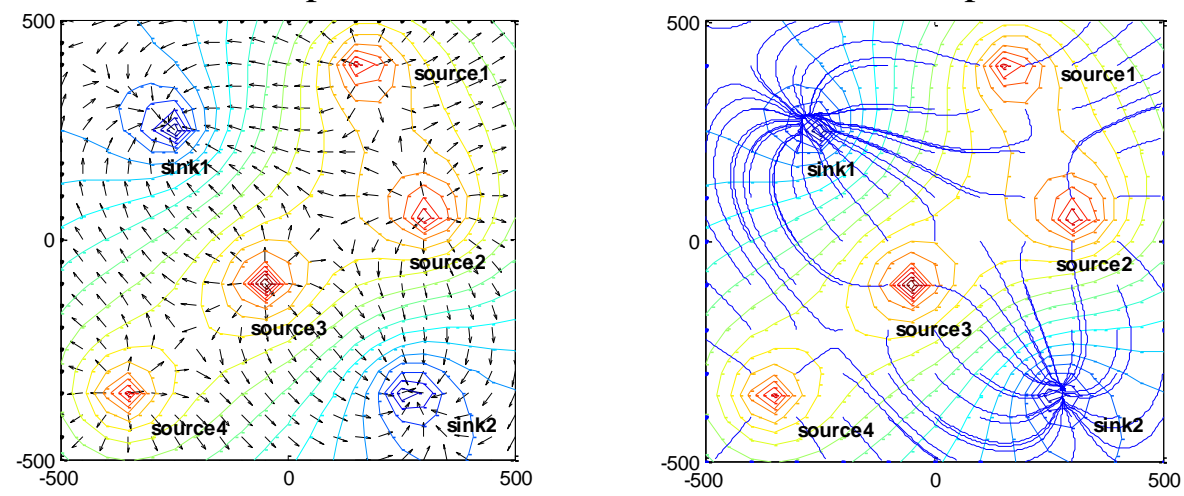

Figure 4. Multiple-node Routing Model

It can be seen from Fig. 4 that the load flow field built with the viscous fluid model shows the load flow direction in the sensor network. Source nodes are in the positions of $(-400,-400),(0,-100),(300$, $100)$ and $(200,400)$, and destination nodes are in the positions of $(250,-300)$ and $(-250,300)$, consistent with original definitions. Loads flow in equilibrium directions without any disorderly flow. Data packets flow from four source nodes to destination nodes orderly in load flow directions.

Fig. 5 shows the comparison of inviscid-fluid-based and viscous-fluid-based multiple-source-node routing models.

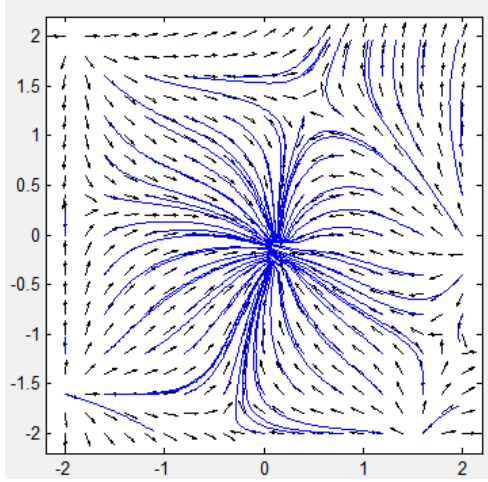

(a) Load Flow Field of Inviscid Fluid

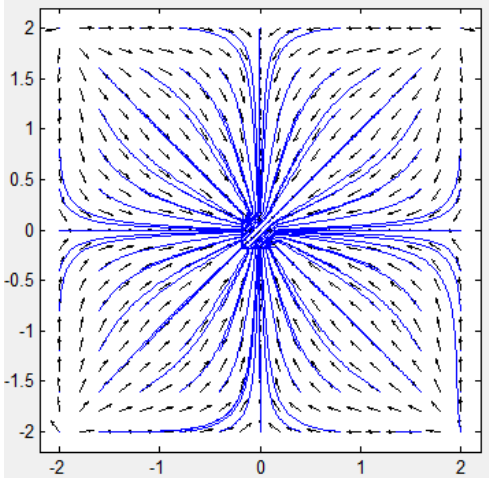

(b) Load Flow Field of Viscous Fluid

Figure 5. Comparison of Multiple Source Nodes 
Fig. 5 (a) shows a simulation figure based on an inviscid-fluid model analysis, and Fig. 5 (b) is a simulation figure obtained from a viscous-fluid model. It can be seen that in Fig. 5 (a), load flow directions are messy on the sink nodes, which may cause the disordered transmission of data packets near destination nodes or the excessive use of some node; while, the load flow field built based on the viscous fluid model solves the problem very well, in which loads flow orderly and evenly without the status of disordered flow.

\section{Conclusion}

The paper introduces the basic theories of fluid mechanics and introduces the fluid mechanics into the analysis on the routing of wireless sensor network. Analysis results show that the flow of viscous fluid is very similar to the flow of data packets in the wireless sensor network, so the paper proposes a wireless sensor network routing model based on fluid mechanics. The result of comparison between inviscid fluid and viscous fluid shows that the viscous fluid is more suitable for the routing analysis of wireless sensor network. Therefore, the paper builds a wireless sensor routing model of N-S equation based on viscous fluid. A key problem in the model is to calculate the density distribution, so the paper builds a density distribution function based on the least square method. Finally, the paper makes the simulation and comparative analysis on the wireless sensor network routing model based on viscous fluid, and finds that the loads in viscous-fluid-based wireless sensor network rouging model flow orderly in an equilibrium state.

\section{References}

[1] N. Jiang, An important fundamental concept of fluid mechanics, Mechanics in Engineering, 37(2015)119-122.

[2] J. J. Chattot and M. M. Hafez, Viscous Fluid Flow and Laminar Boundary Layers, Theoretical and Applied Aerodynamics. Springer Netherlands, 2015,261-291.

[3] R. A. Bagnold, Experiments on the gravity-free dispersion of large solid spheres in a Newtonian Fluid under shear, The Physics of Sediment Transport by Wind and Water, ASCE, 2015,49-63.

[4] A. D. Rosis, Harmonic oscillations of laminae in non-Newtonian fluids: A lattice boltzmann-immersed boundary approach, Advances in Water Resources, 73(2014)97-107.

[5] Z. P. Jiang, Z. Y. Chen, H. Yan and W. Zhao, Study on multipath routing protocols in wireless sensor networks based on potential flow theory, Chinese Journal of Sensors and Actuators, 2015, 28(2015)1873-1878.

[6] Y. Wang and Y. W. Yang, Sensor network deployment technology based on flow control model, Computer Engineering, 37(2011) 80-83.

[7] X. S. Shi, H. Feng, T. Yang and B. Hu, Data selection and recovery based on a customized discrete laplace operator in wireless sensor networks, Journal of Chinese Computer Systems, 31(2016)65-70.

[8] H. Wang and Y. Q. Huang, Smoothing and compression algorithm of triangular mesh models based on laplacian, Computer Systems and Applications, 24(2015) 191-195.

[9] Y. P. Lai, Y. J. Zhou and H. W. Ding, et. Variational learning for finite inverted dirichlet mixture models and applications, Acta Electronica Sinica, 42(2014)1435-1440.

[10] Lupe. On the geometry of systems of first order partial differential equations, Journal of High Energy Physics, 2015(2014) 1-27. 